\title{
Pretreatment clinical stage predicts locoregional recurrence in patients with esophageal cancer who achieved a complete clinical response to chemoradiotherapy
}

\author{
Pei-Xuan Liw, MD, ${ }^{\text {a }}$ Yu-Wen Wen, PhD,,${ }^{\text {a,b }}$ Chang-Yo Tsai, MD, ${ }^{a}$ Hsien-Kun Chang, MD, ${ }^{c}$ \\ Chen-Kan Tseng, MD, ${ }^{\mathrm{d}}$ Tsung-Min Hung, MD, ${ }^{\mathrm{d}}$ and Yin-Kai Chao, MD
}

\section{ABSTRACT}

Objective: Definitive chemoradiotherapy (dCRT) represents a curative nonsurgical treatment option for patients with esophageal cancer. However, tumor recurrence is common after dCRT, even when clinical complete response (cCR) is achieved. Here, we investigated the timing, patterns, and risk factors for recurrence in patients with esophageal squamous cell carcinoma (ESCC) who achieved cCR following dCRT.

Methods: We retrospectively examined the clinical records of patients with ESCC who achieved cCR following dCRT between 2001 and 2014. Locoregional recurrence (LR) was defined as a recurrence occurring in the esophageal lumen and/or locoregional lymph nodes. Recurrences at any other sites were considered as distant recurrences (DRs).

Results: A total of 102 patients who achieved cCR were included. After a mean follow-up of 54.5 months, 51 patients developed recurrences (34 LRs, 6 combined LR and DR, and $11 \mathrm{DRs}$ ). The cumulative 1-, 3-, and 5-year recurrence rates were $35 \%, 46 \%$, and $50 \%$, respectively. The mean time to recurrence for the 40 patients with LRs (including LRs plus LRs/DRs) was significantly shorter (281.4 days) compared with that of patients with DRs (643.6 days; $P=.006$ ), with $95 \%$ of the former being diagnosed within 2 years. Multivariate Cox regression analysis identified pretreatment clinical stage III as the only independent risk factor for LR (hazard ratio, 2.732; 95\% confidence interval; 1.063-7.020; $P=.037)$.

Conclusions: Disease recurrence occurs in $50 \%$ of ESCC patients who achieve cCR following dCRT, with LR being the most common pattern. Advanced pretreatment clinical stage is an independent risk factor for LR. (J Thorac Cardiovasc Surg 2018;155:2233-42)

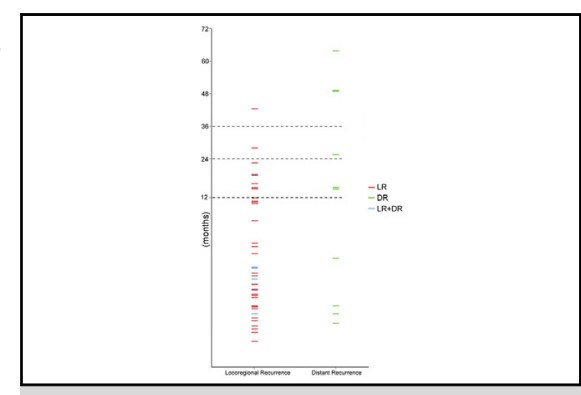

Time interval between the diagnosis of clinical complete response and disease recurrence.

\section{Central Message}

Disease recurrence occurs in $50 \%$ of patients with esophageal squamous cell carcinoma who achieve clinical complete response following definitive chemoradiotherapy.

Perspective

Disease recurrence occurs in $50 \%$ of patients with esophageal squamous cell carcinoma who achieve clinical complete response following definitive chemoradiotherapy, with locoregional recurrence being the most common pattern. Advanced pretreatment clinical stage is an independent risk factor for locoregional recurrence.

See Editorial Commentary page 2243
Esophageal carcinoma is a highly lethal malignancy characterized by dismal clinical outcomes. ${ }^{1}$ When patients with locally advanced esophageal cancer are unfit to receive

\footnotetext{
From the Divisions of ${ }^{\mathrm{a}}$ Thoracic Surgery and ${ }^{\mathrm{c}} \mathrm{Hematology} /$ Oncology, ${ }^{\mathrm{d}}$ Department of Radiation Oncology, Chang Gung Memorial Hospital-Linko, and ${ }^{\mathrm{b}}$ Clinical Informatics and Medical Statistics Research Center, Chang Gung University, Taoyuan, Taiwan.

Supported by grant No. CMRPG3G0291 from the Chang Gung Memorial Hospital, Taoyuan, Taiwan.

Received for publication Oct 31, 2016; revisions received Sept 16, 2017; accepted for publication Dec 17, 2017; available ahead of print Feb 3, 2018.

Address for reprints: Yin-Kai Chao, MD, Division of Thoracic Surgery, Chang Gung

Memorial Hospital-Linko, Taoyuan, Taiwan (E-mail: chaoyk@cgmh.org.tw). $0022-5223 / \$ 36.00$

Copyright (c) 2017 by The American Association for Thoracic Surgery

https://doi.org/10.1016/j.jtcvs.2017.12.082
}

surgery or otherwise decline it, definitive chemoradiotherapy (dCRT) is generally regarded as the most suitable alternative treatment option with curative intent, especially in esophageal squamous cell carcinoma (ESCC). ${ }^{2-4}$ After dCRT, at least $30 \%$ to $40 \%$ of ESCC patients do achieve clinical complete response (cCR), defined as the absence of residual cancer on endoscopic biopsy, computed

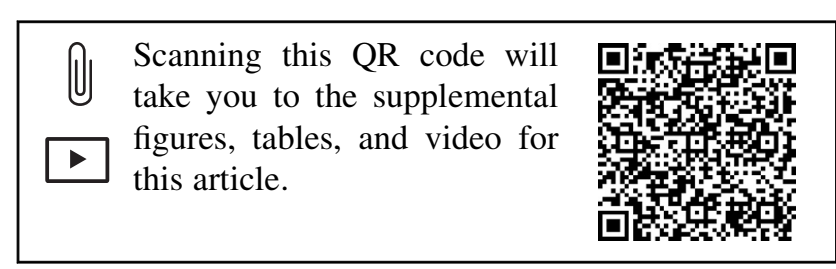




\section{Abbreviations and Acronyms \\ $\mathrm{cCR}=$ clinical complete response \\ $\mathrm{CR}=$ complete response \\ $\mathrm{CT}=$ computed tomography \\ $\mathrm{dCRT}=$ definitive chemoradiotherapy \\ $\mathrm{ESCC}=$ esophageal squamous cell carcinoma \\ $\mathrm{LR}=$ local recurrences \\ nCRT $=$ neoadjuvant chemoradiotherapy \\ PET $=$ positron-emission tomography}

tomography (CT), and/or positron-emission tomography (PET) ${ }^{5-7}$ Despite the lack of clinical evidence of residual cancer, tumor recurrences after the achievement of cCR are not uncommon (reported recurrence rate, 40\%). ${ }^{8,9}$ The high recurrence rates have been generally attributed to the low accuracy of the diagnostic methods used to confirm the presence or absence of residual cancer. ${ }^{10-12}$ Some patients who actually harbor residual disease following dCRT can erroneously be diagnosed as having achieved cCR, ultimately experiencing disease recurrence. In this scenario, better diagnostic modalities and optimized surveillance strategies are paramount for increasing the likelihood of a timely and successful salvage treatment. Starting from these assumptions, we designed the current study to investigate the timing and patterns of disease recurrences in a series of 102 patients with ESCC who achieved cCR following dCRT. The identification of patients at high risk of recurrence may potentially improve their clinical management and optimize their follow-up schedules.

\section{MATERIALS AND METHODS \\ Patient Population}

We retrospectively reviewed the clinical records of patients with locally advanced ESCC who had undergone dCRT at the Chang Gung Memorial Hospital, Taoyuan, Taiwan, between January 2001 and October 2014. Patients who were coded as having achieved cCR $(24.4 \%)$ in the dataset were initially selected. Subsequently, our esophageal cancer multidisciplinary team carefully re-reviewed all of the clinical data using the cCR criteria listed below. Only patients for whom consensus was reached on the achievement of cCR were included. The following exclusion criteria were applied: treatment with an insufficient radiation dose (ie, < $50 \mathrm{~Gy}$ ), ${ }^{13}$ unavailability of upper endoscopy or image data for rereview, and follow-up examinations performed outside of our institution. Pretreatment staging and restaging were based on CT of the chest and abdomen, esophagography, and endoscopic ultrasonography performed with an ultrasonic miniprobe (UM2R/12 MHz or UM3R/20 MHz; Olympus Inc, Tokyo, Japan). Patients were staged according to the 2010 American Joint Committee on Cancer staging criteria, seventh edition. Patient performance status was assessed by using the Eastern Cooperative Oncology Group scale, which ranges from 0 (fully active, able to carry on all predisease activities without restriction) to 5 (dead). ${ }^{14}$ The severity of comorbidities was determined using the Charlson comorbidity index, a measure of the overall disease burden based on the presence of 19 distinct
TABLE 1. General characteristics of the study patients

\begin{tabular}{|c|c|}
\hline Parameter & Result \\
\hline No. of patients & 102 \\
\hline Age $(y)$ & $60.36 \pm 12.02$ \\
\hline \multicolumn{2}{|l|}{ Sex } \\
\hline Male & $96(94.1)$ \\
\hline Female & $6(5.9)$ \\
\hline \multicolumn{2}{|l|}{ Eastern Cooperative Oncology } \\
\hline \multicolumn{2}{|l|}{ Group scale } \\
\hline 0 & $9(8.8)$ \\
\hline 1 & $72(70.6)$ \\
\hline 2 & $21(20.6)$ \\
\hline \multicolumn{2}{|l|}{ Charlson comorbidity index } \\
\hline 0 & $58(56.9)$ \\
\hline 1 & $28(27.5)$ \\
\hline$>1$ & $16(15.7)$ \\
\hline \multicolumn{2}{|l|}{ Clinical $\mathrm{T}$ stage } \\
\hline 2 & $25(24.5)$ \\
\hline 3 & $46(45.1)$ \\
\hline 4 & $31(30.4)$ \\
\hline \multicolumn{2}{|l|}{ Pretreatment clinical $\mathrm{N}$ stage } \\
\hline 0 & $11(10.8)$ \\
\hline 1 & $45(54.9)$ \\
\hline 2 & $29(28.4)$ \\
\hline 3 & $6(5.9)$ \\
\hline \multicolumn{2}{|l|}{ Pretreatment clinical stage } \\
\hline II & $26(25.5)$ \\
\hline III & $76(74.5)$ \\
\hline Tumor length $(\mathrm{cm})$ & $6.11 \pm 2.76$ \\
\hline \multicolumn{2}{|l|}{ Tumor location } \\
\hline Upper third & $37(36.3)$ \\
\hline Middle third & $50(49)$ \\
\hline Lower third & $15(14.7)$ \\
\hline \multicolumn{2}{|l|}{ Chemotherapy } \\
\hline 5-fluorouracil plus cisplatin & $99(97.1)$ \\
\hline Carboplatin plus paclitaxel & $3(2.9)$ \\
\hline \multicolumn{2}{|l|}{ Radiation dose } \\
\hline 60 Gy & $95(93.1)$ \\
\hline$>60 \mathrm{~Gy}$ & $7(6.9)$ \\
\hline
\end{tabular}

Values are presented as $\mathrm{n}$, mean \pm standard deviation, or $\mathrm{n}(\%)$.

medical disease categories. Each comorbidity is weighted (from 1 to 6) according to the extent to which it predicts mortality. ${ }^{15}$ Because of the retrospective nature of the study, the Institutional Review Board of the Chang Gung Memorial Hospital waived the requirement for informed consent.

\section{dCRT}

Two chemotherapy regimens were used throughout the study period. The first consisted of 5 -fluorouracil $\left(1000 \mathrm{mg} / \mathrm{m}^{2} / \mathrm{d}\right)$ plus cisplatin $\left(75 \mathrm{mg} / \mathrm{m}^{2}\right)$ repeated every 3 weeks for a total of 3 to 4 cycles, whereas the second was based on the combination of carboplatin (doses titrated to achieve an area under the curve of $2 \mathrm{mg} / \mathrm{mm} / \mathrm{min}$ ) and paclitaxel $\left(50 \mathrm{mg} / \mathrm{m}^{2}\right.$ of body surface area) repeated every week for a total of 5 to 6 cycles. Concurrent radiotherapy was delivered either at a dose of $2 \mathrm{~Gy} /$ $\mathrm{d}, 5 \mathrm{~d} / \mathrm{wk}$, for a total dose of $60 \mathrm{~Gy}$ in 30 fractions or $1.8 \mathrm{~Gy} / \mathrm{d}, 5 \mathrm{~d} / \mathrm{wk}$, for a total dose of $63 \mathrm{~Gy}$ in 35 fractions. 
TABLE 2. Detailed characteristics of the 51 patients who developed recurrences

\begin{tabular}{|c|c|c|c|c|c|}
\hline Patient no. & $\begin{array}{c}\text { Time to } \\
\text { recurrence }(d)\end{array}$ & $\begin{array}{c}\text { Local } \\
\text { recurrence }\end{array}$ & $\begin{array}{c}\text { Regional lymph node } \\
\text { recurrence }\end{array}$ & $\begin{array}{c}\text { Distant recurrence } \\
\text { (anatomic site) }\end{array}$ & Treatment \\
\hline 1 & 60 & + & - & - & Salvage esophagectomy \\
\hline 2 & 60 & + & - & - & Salvage esophagectomy \\
\hline 3 & 78 & + & - & - & Salvage esophagectomy \\
\hline 4 & 85 & + & - & - & Chemotherapy \\
\hline 5 & 92 & + & - & - & Salvage esophagectomy \\
\hline 6 & 92 & + & - & - & None \\
\hline 7 & 102 & + & - & - & None \\
\hline 8 & 108 & + & - & - & Chemotherapy \\
\hline 9 & 128 & + & - & - & None \\
\hline 10 & 132 & + & + & - & Chemoradiotherapy \\
\hline 11 & 133 & + & - & - & Chemotherapy \\
\hline 12 & 152 & + & + & - & Chemotherapy \\
\hline 13 & 156 & + & - & - & Chemotherapy \\
\hline $14^{*}$ & 158 & + & - & - & Chemotherapy \\
\hline 15 & 168 & + & - & - & Salvage esophagectomy \\
\hline 16 & 180 & + & - & - & Chemotherapy \\
\hline 17 & 197 & + & - & - & Salvage esophagectomy \\
\hline $18^{*}$ & 204 & + & - & - & ESD \\
\hline 19 & 244 & + & - & - & Salvage esophagectomy \\
\hline $20^{*}$ & 262 & + & - & - & Salvage esophagectomy \\
\hline 21 & 268 & + & - & - & Salvage esophagectomy \\
\hline 22 & 314 & + & - & - & Chemotherapy \\
\hline 23 & 350 & + & - & - & None \\
\hline 24 & 352 & + & - & - & Salvage esophagectomy \\
\hline 25 & 353 & + & - & - & Chemotherapy \\
\hline 26 & 357 & + & - & - & Salvage esophagectomy \\
\hline 27 & 383 & + & - & - & Chemotherapy \\
\hline $28 *$ & 400 & + & - & - & Salvage esophagectomy \\
\hline 29 & 447 & + & - & - & Chemotherapy \\
\hline $30^{*}$ & 448 & + & - & - & Chemotherapy \\
\hline 31 & 543 & + & - & - & Salvage esophagectomy \\
\hline $32 *$ & 674 & + & - & - & Chemotherapy \\
\hline 33 & 839 & - & + & - & Celiac lymph node dissection \\
\hline $34 *$ & 1273 & + & - & - & Chemotherapy \\
\hline 35 & 117 & + & - & + (Liver, bone, lung $)$ & Chemotherapy \\
\hline 36 & 167 & + & - & $+($ Bone $)$ & Chemoradiotherapy \\
\hline 37 & 191 & + & + & $+($ Mesenteric LN) & No treatment \\
\hline 38 & 214 & + & + & $+($ Paraaortic LN $)$ & Chemotherapy \\
\hline 39 & 217 & + & + & + (Kidney) & Chemotherapy \\
\hline 40 & 556 & + & - & $+($ Neck LN $)$ & No treatment \\
\hline 41 & 94 & - & - & $+($ Lung $)$ & Chemotherapy \\
\hline 42 & 96 & - & - & $+($ Neck LN $)$ & Chemoradiotherapy \\
\hline 43 & 114 & - & - & $+($ Neck LN $)$ & Neck LN dissection \\
\hline 44 & 132 & - & - & $+($ Neck LN, liver $)$ & No treatment \\
\hline
\end{tabular}


TABLE 2. Continued

\begin{tabular}{lccccc}
\hline Patient no. & $\begin{array}{c}\text { Time to } \\
\text { recurrence }(\mathbf{d})\end{array}$ & $\begin{array}{c}\text { Local } \\
\text { recurrence }\end{array}$ & $\begin{array}{c}\text { Regional lymph node } \\
\text { recurrence }\end{array}$ & $\begin{array}{c}\text { Distant recurrence } \\
\text { (anatomic site) }\end{array}$ & Treatment \\
\hline 45 & 234 & - & - & + (Bone) & No treatment \\
46 & 384 & - & - & + (Brain) & Resection of brain metastases \\
47 & 403 & - & - & + (Neck LN, lung) & Chemotherapy \\
$48^{*}$ & 768 & - & - & + (Paraaortic LN) & Chemotherapy \\
49 & 1469 & - & - & + (Brain) & Resection of brain metastases \\
50 & 1472 & - & - & + (Pleural metastases) & No Treatment \\
\hline 51 & 1914 & - & 6 & Resection of lung metastases \\
\hline
\end{tabular}

$E S D$, Endoscopic submucosal dissection; $L N$, lymph node. *Patient alive at the time of analysis.

\section{Assessment of Treatment Response}

Patient restaging was planned at 8 to 12 weeks after the completion of dCRT. Posttreatment assessments consisted of a thorough physical examination, endoscopic biopsies, CT examinations of the thorax and abdomen, and (as of 2007) PET imaging.

All endoscopic evaluations were performed by 3 experienced gastroenterologists. Patients were deemed to have achieved cCR when both endoscopic and radiologic complete response (CR) was evident. Endoscopic $\mathrm{CR}$ (on upper endoscopy) was diagnosed according to the Japanese endoscopic criteria, whereas the presence of radiologic CR on CT was defined according to the revised Response Evaluation Criteria In Solid Tumors 1.1 criteria. ${ }^{9} 16$ Endoscopic CR was considered to be present when the tumor was no longer evident, the ulcerative lesion (slough) disappeared, and cancer cells in biopsy specimens were absent. If any suspicious lesions were identified on upper endoscopy following dCRT, a minimum of 3 biopsy samples were acquired to determine whether tumor cells were present. In the absence of identifiable lesions on upper endoscopy, random biopsies were acquired from the previous tumor site. Radiologic $\mathrm{CR}$ of an affected lymph node was defined as its shrinkage to $<10 \mathrm{~mm}$ in the short axis on CT imaging. PET-CR was defined as a decrease in fluorodeoxyglucose uptake in the primary tumor and affected lymph nodes to a level that was indistinguishable from the surrounding normal tissue. Diffuse fluorodeoxyglucose accumulation in the field of radiotherapy in the absence of focal activity was considered radiotherapy-induced esophagitis and classified as PET-CR. Patients who were judged not to have achieved CR after dCRT were offered salvage surgery if their conditions allowed.

\section{Posttreatment Surveillance and Late Toxicity Assessment}

The posttreatment surveillance protocol consisted of follow-up visits (every 3 months during the first 2 years, every 6 months during the third and fourth years, and every 6-12 months thereafter). Contrast-enhanced CT scans were scheduled every 6 to 8 months during the first 2 years and on an annual basis thereafter. Upper endoscopy was not routinely performed in cCR patients unless clinically indicated. Reminders were issued via telephone calls to re-engage patients who had missed a scheduled follow-up appointment. Noncompliance with the surveillance protocol was defined as missing 2 or more consecutive clinic visits or missing at least $1 \mathrm{CT}$ scan at any time during the course of the first 2 years of follow-up.

\section{Definition of Recurrence and Survival}

The patterns of treatment failure were classified according to their first site of recurrence. Locoregional recurrence (LR) was defined as a recurrence that occurred in the esophageal lumen or locoregional lymph nodes. Recurrences at any other site were considered distant recurrences (DRs).
When metastases occurred in patients with a second primary cancer at sites other than the esophagus, the origins of the metastatic lesions were determined according to their distributions and/or histologic findings. Overall survival (OS) was calculated from the date of cCR to the date of death (or otherwise censored on December 31,2016). The time to recurrence was defined as the interval between the date of $\mathrm{cCR}$ and the date of recurrence. Survival after recurrence was calculated from the time of recurrence to the end of the follow-up period.

\section{Statistical Analysis}

Patients with esophageal cancer frequently have comorbid conditions and might die of noncancer-related causes, which can pose as competing risks for recurrences. Therefore, a competing risk model was constructed to summarize the cumulative incidences of recurrence. Univariate and multivariable Cox proportional hazard analyses were used to identify factors associated with LRs. The following variables were analyzed in the model: age, sex, Eastern Cooperative Oncology Group scale status, tumor length, tumor location, clinical $\mathrm{T}$ stage, clinical $\mathrm{N}$ stage, overall clinical stage, radiotherapy dose (>6000 cGy vs $\leq 6000$ cGy), chemotherapy regimen, and chemotherapy dose (originally planned dose vs below the originally planned dose). Simple univariate models were constructed; factors that were associated with LR with a $P$ value $<.1$ (ie, age, clinical $\mathrm{T}$ stage, and clinical stage) were then entered as covariates into the multivariable model. A backward selection procedure was used to identify the independent risk factors for LR. The results of regression analyses are expressed as hazard ratios and their $95 \%$ confidence intervals. Survival curves in patients with and without recurrence were plotted using the Kaplan-Meier method and compared with the log-rank test. All calculations were performed with SPSS statistical software version 22.0 (IBMSPSS Inc, Armonk, NY) and the mstate library in R (R Foundation for Statistical Computing, Vienna, Austria). ${ }^{17}$

\section{RESULTS \\ Patient Characteristics}

A study flowchart is provided in Figure E1. Of 505 consecutive ESCC patients who were treated with dCRT between January 2001 and October 2014, 128 (24.4\%) achieved cCR. When cCR cases were identified, the members of our esophageal multidisciplinary team performed a careful re-review of all clinical data (blinded to survival outcomes). A total of 26 patients were excluded for the following reasons: incomplete image data for CT and/or upper endoscopy for the additional re-review $(n=18)$, incomplete treatment details $(n=6)$, and incomplete follow-up 


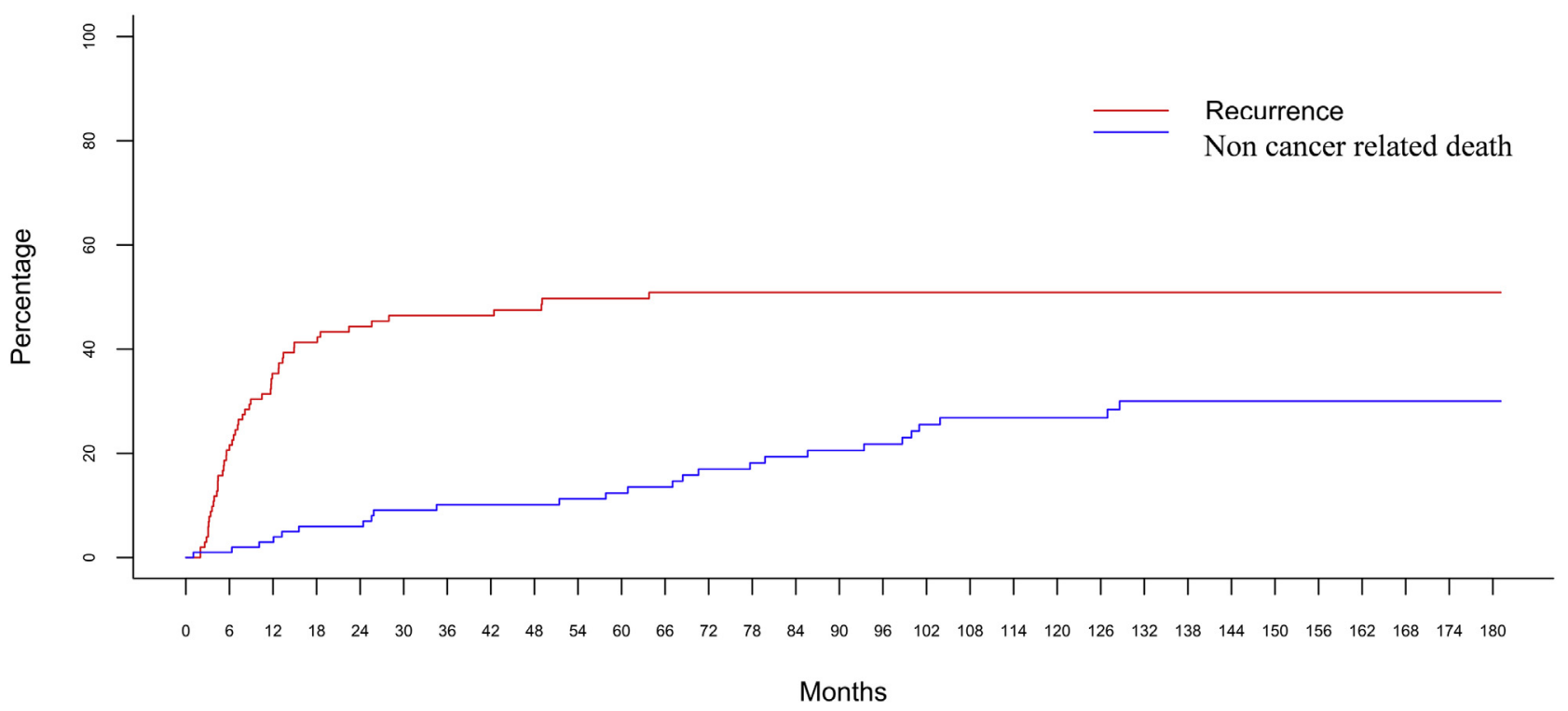

FIGURE 1. Cumulative incidence of recurrences and noncancer-related deaths in a competing risk setting.

data $(\mathrm{n}=2)$. Ultimately, the final study cohort comprised 102 patients (mean age, 60.4 years; age range, $37-83$ years) who completed dCRT. The general characteristics of the study patients are summarized in Table 1 . Before treatment, the $\mathrm{T}$ stages were $\mathrm{T} 2$ in $24.5 \%$ of patients, $\mathrm{T} 3$ in $45.1 \%$ of patients, and $\mathrm{T} 4$ in $30.4 \%$ of patients. Of all patients, 91 $(89.2 \%)$ had clinical evidence of lymph node metastasis. The primary tumor was located in the upper third of the esophagus in $36.3 \%$ of patients, in the middle third in $49 \%$, and in the lower third in $14.7 \%$. The mean pretreatment tumor length based on esophagography findings was $6.11 \mathrm{~cm}$ (range, 1-16 cm). With regard to chemotherapy administration, most patients $(97.1 \%)$ received the 5-fluorouracil plus cisplatin regimen; 8 patients did not receive the full dose as planned in the protocol.

The diagnosis of cCR was confirmed through upper endoscopy and CT in 88 patients and via 3 different diagnostic modalities (ie, upper endoscopy, CT, and PET) in the remaining 14 patients.

\section{Survival and Patterns of Recurrence}

The mean follow-up time was 54.5 months (median, 31 months; interquartile range, 14.9-95.3 months). Ninety-eight patients were considered compliant to the

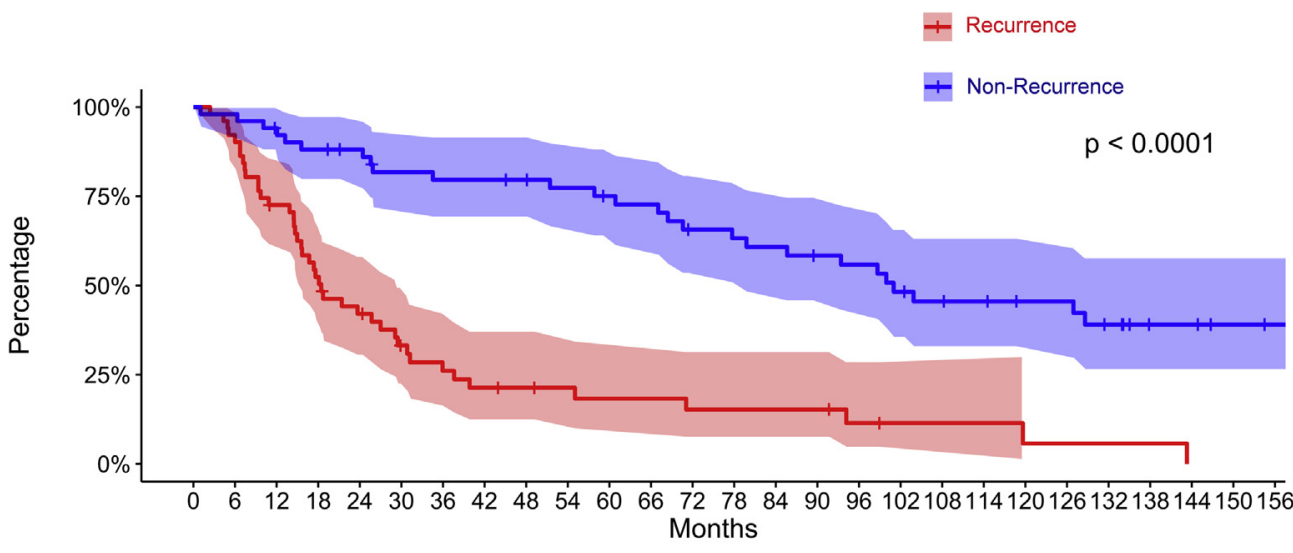

Number at risk by time

Recurrence $-\begin{array}{llllllllllllllllllllllllllll}51 & 47 & 36 & 26 & 20 & 14 & 11 & 9 & 8 & 7 & 6 & 6 & 5 & 5 & 5 & 5 & 3 & 2 & 2 & 2 & 1 & 1 & 1 & 1 & 0 & 0 & 0\end{array}$ Non-Recurrence \begin{tabular}{rlllllllllllllllllllllllllllllllll}
51 & 50 & 47 & 44 & 42 & 38 & 37 & 37 & 36 & 34 & 32 & 31 & 27 & 26 & 25 & 23 & 22 & 19 & 17 & 16 & 14 & 14 & 11 & 7 & 7 & 5 & 4 \\
\hline
\end{tabular}

$\begin{array}{llllllllllllllllll}0 & 6 & 12 & 18 & 24 & 30 & 36 & 42 & 48 & 54 & 60 & 6 & 72 & 78 & 84 & 90 & 96 & 102108114120126132138144150156 \\ \text { Months }\end{array}$

FIGURE 2. Overall survival of patients with and without disease recurrence. 


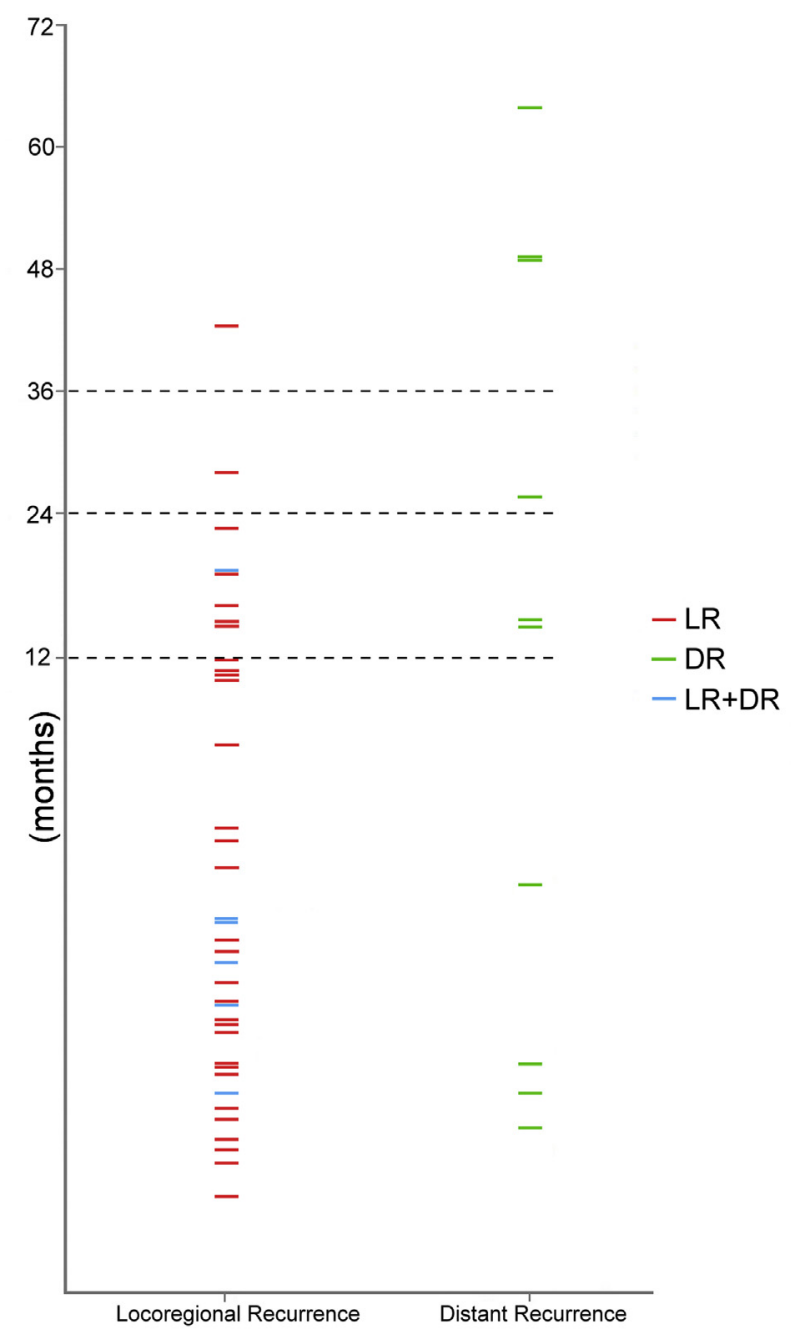

FIGURE 3. Durations between the diagnosis of clinical complete response and locoregional recurrence $(L R)$, distant recurrence $(D R)$, and combined locoregional and distant recurrence $(L R D R)$.

surveillance program, whereas the remaining 4 patients either attended follow-up sessions occasionally or never presented. Sixty-nine patients were dead at the time of analysis. The 5-year OS rate was $46.1 \%$, with a median OS of 51.4 months.

Fifty-one patients $(50 \%)$ experienced disease recurrences. LR alone, combined LR and DR, and DR alone were diagnosed in 34,6 , and 11 patients, respectively. Table 2 shows the sites of first recurrence, as well as the treatment approaches implemented after recurrence in this patient group. Among them, 43 recurrence sites were identified by histopathology (endoscopic biopsy in 36 patients and percutaneous or surgical biopsy in 7 cases), whereas the remaining 8 cases were discovered during follow-up. The cumulative incidence estimators for both competing events; that is, recurrences and noncancer-related deaths, are shown in Figure 1. The cumulative incidence estimators of recurrence by the competing risk method at $1,2,3,4$, and 5 years were $35 \%, 44 \%, 46 \%, 47 \%$, and $50 \%$, respectively. The cumulative incidence of noncancer-related death summarizes the probability of dying before recurrence; if death occurs first, the observation is not considered censored in the competing risk approach, although it still contributes to the competing event of death.

The median OS was significantly lower in patients with recurrences (18.4 months) compared with those without (101 months, $P<.001)$ (Figure 2). The mean time interval between the diagnosis of $\mathrm{cCR}$ and the detection of recurrence was 359.5 days (median, 214 days; range, 601914 days). The time to recurrence of the 40 patients with LR (including both patients with LR alone and those with both LR and DR) was significantly shorter (mean, 281.4 days) compared with those of patients with DR (mean, 643.6 days; $P=.006$ ). Notably, more than $95 \%$ of all LRs occurred within 2 years, compared with 4 years for $90 \%$ of DRs. The details of the time intervals between the diagnoses of cCR and LR, combined LR and DR, and DR are shown in Figure 3. Notably, the introduction of PET for the confirmation of cCR did not modify the recurrence rate (cCR with PET vs cCR without PET: $57.1 \%$ vs $48.9 \%$, respectively; $P=.775$ ), with LR remaining the most common failure pattern.

\section{Management of Recurrences and Survival After Recurrence}

Of 34 patients who experienced LRs alone, 15 were treated with potentially curative intent (salvage esophagectomy [13 patients], endoscopic submucosal dissection [1 patient], and resection of celiac lymph nodes [1 patient]), 15 patients received chemotherapy/chemoradiotherapy (including 4 patients who refused salvage esophagectomy), and 4 received supportive care only. Of 13 patients who underwent salvage esophagectomy, R0 resection was successfully achieved in 6 . One patient had a microscopically incomplete (R1) resection, whereas macroscopically incomplete (R2) resections were noted in the remaining 6 patients (because of tumor invasion in the airways in 4 patients and in the aorta in 2 patients). Inhospital mortality occurred in 2 . Of 6 patients who developed combined LR and DR, 4 were treated with chemotherapy/chemoradiotherapy and 2 received supportive care. Of 11 patients with DR alone, 4 underwent surgical resection of distant metastases ( 2 in the brain [ 1 for symptom control] and 1 each in the lung and neck lymph node), 4 were treated with chemotherapy or chemoradiotherapy, and 3 received supportive care only. Among 51 patients with recurrences, 8 (patients 14, 18, 20, 28, 30, 32, 34, and 48; 7 with LR and 1 with DR) were still alive at the end of the study. The median OS after recurrence was 10.2 months (interquartile range, 3-14 months), with the type of recurrence (LRs vs combined LR and DR vs 
TABLE 3. Results of univariate and multivariable Cox regression analysis for risk factors of locoregional recurrence

\begin{tabular}{|c|c|c|c|c|}
\hline Risk factor & $\begin{array}{c}\text { Univariate } \\
\text { Hazard ratio }(95 \% \text { confidence } \\
\text { interval) }\end{array}$ & $P$ value & $\begin{array}{c}\text { Multivariable } \\
\begin{array}{c}\text { Hazard ratio }(95 \% \text { confidence } \\
\text { interval) }\end{array}\end{array}$ & $P$ value \\
\hline Age (y) & $0.970(0.946-0.996)$ & .023 & $0.975(0.949-1.001)$ & .055 \\
\hline $\begin{array}{l}\text { Sex } \\
\qquad \text { Male } \\
\text { Female }\end{array}$ & $\begin{array}{c}\text { Reference } \\
1.452(0.447-4.717)\end{array}$ & .535 & N/A & \\
\hline $\begin{array}{c}\text { ECOG scale } \\
0 \\
1 \\
2\end{array}$ & $\begin{array}{c}\text { Reference } \\
0.840(0.295-2.393) \\
0.679(0.191-2.415)\end{array}$ & $\begin{array}{l}.745 \\
.549\end{array}$ & & \\
\hline Tumor length $(\mathrm{cm})$ & $1.036(0.928-1.158)$ & .525 & N/A & \\
\hline $\begin{array}{l}\text { Location } \\
\text { U } \\
\text { M } \\
\text { L }\end{array}$ & $\begin{array}{c}\text { Reference } \\
1.087(0.549-2.153) \\
1.089(0.418-2.835)\end{array}$ & $\begin{array}{l}.811 \\
.862\end{array}$ & N/A & \\
\hline $\begin{array}{l}\text { Clinical T stage } \\
2 \\
3 \text { or } 4\end{array}$ & $\begin{array}{c}\text { Reference } \\
2.418(1.014-5.767)\end{array}$ & .046 & $\begin{array}{c}\text { Reference } \\
1.181(0.362-3.859)\end{array}$ & .783 \\
\hline $\begin{array}{l}\text { Clinincal N stage } \\
0 \\
1 \\
2 \text { or } 3\end{array}$ & $\begin{array}{c}\text { Reference } \\
3.389(0.804-14.290) \\
2.474(0.553-11.069)\end{array}$ & $\begin{array}{l}.096 \\
.236\end{array}$ & N/A & \\
\hline $\begin{array}{l}\text { Clinical stage } \\
\text { II } \\
\text { III }\end{array}$ & $\begin{array}{c}\text { Reference } \\
3.020(1.181-7.718)\end{array}$ & .021 & $\begin{array}{c}\text { Reference } \\
2.732(1.063-7.020)\end{array}$ & .037 \\
\hline $\begin{array}{l}\text { Radiotherapy dose (cGy) } \\
\quad \leq 6000 \\
\quad>6000\end{array}$ & $\begin{array}{c}\text { Reference } \\
1.124(0.346-3.649)\end{array}$ & .845 & N/A & \\
\hline $\begin{array}{l}\text { Chemotherapy } \\
\text { PF regimen } \\
\text { PC regimen }\end{array}$ & $\begin{array}{c}\text { Reference } \\
0.901(0.124-6.576)\end{array}$ & .918 & N/A & \\
\hline $\begin{array}{l}\text { Chemotherapy dose } \\
\text { Planned dose } \\
<\text { Planned dose }\end{array}$ & $\begin{array}{c}\text { Reference } \\
1.896(0.742-4.845)\end{array}$ & .181 & & \\
\hline
\end{tabular}

$E C O G$, Eastern Cooperative Oncology Group; $N / A$, not applicable; $U$, upper; $M$, middle; $L$, lower; $P F$, cisplatin +5 -fluorouracil; $P C$, paclitaxel + carboplatin.

DRs) not having a significant influence on survival $(P=.3)$ (Figure 3).

\section{Factors Associated With Recurrences}

Risk factors for LRs and DRs were analyzed separately. Univariate Cox regression analysis revealed that LR was significantly associated with patient age and clinical stage. Only clinical stage retained its independent significance after adjustment for potential confounders in multivariable analysis (hazard ratio, 2.732; 95\% confidence interval, $1.063-7.020 ; P=.037$ ) (Table 3). Age was identified as an independent risk factor for DR (Table E1).

\section{DISCUSSION}

The optimal management approach for patients with ESCC remains controversial; an increasing number of oncologists currently advocate treating these patients with dCRT, followed by expectant management in patients who achieve cCR and salvage surgery for patients with persistent residual disease. ${ }^{2-4}$ The results of our study show that $50 \%$ of ESCC patients who achieved cCR following dCRT experienced disease recurrences, with LR being the predominant failure pattern. Our results echo those of 2 previously published studies (Table 4)., Accordingly, evidence obtained from patients treated with neoadjuvant chemoradiotherapy (nCRT) indicates that cCR should not be considered as a reliable proxy for complete esophageal cancer eradication. A high percentage $(40 \%$ to $60 \%)$ of patients in whom cCR was considered achieved still had cancer foci in their resected esophageal specimens (non-ypT0) obtained after scheduled resection. If the same assumption is valid for 
TABLE 4. Published literature on recurrences in patients with esophageal cancer after definitive chemoradiotherapy (dCRT)

\begin{tabular}{|c|c|c|c|}
\hline & Ishihara and colleagues $^{6}$ & Adenis and colleagues ${ }^{7}$ & Current study \\
\hline Number of cCR patients & 110 & 110 & 102 \\
\hline Rate of cCR after dCRT (\%) & 40.9 & 27 & 24.4 \\
\hline ESCC diagnosis $(\%)$ & 100 & 93.6 & 100 \\
\hline Radiotherapy dose (Gy) & 60 Gy $(100 \%)$ & $\begin{array}{l}\leq 50.4 \text { Gy }(55.5 \%) \\
>50 \text { Gy }(44.5 \%)\end{array}$ & $\begin{array}{c}60 \text { Gy }(93.1 \%) \\
>60 \text { Gy }(6.9 \%)\end{array}$ \\
\hline Median follow-up time (mo [range]) & $21(4-67)$ & $72(4.8-117.6)$ & $54.5(2.1-181)$ \\
\hline Patients with recurrence (n [\%]) & $28(25.5)$ & $44(40)$ & $51(50)$ \\
\hline $\begin{array}{l}\text { Type of recurrence } \\
\text { LR (LR alone or combined LR and DR) } \\
\text { DR }\end{array}$ & $\begin{array}{r}20 \\
8\end{array}$ & $\begin{array}{c}32(26 / 6) \\
12\end{array}$ & $\begin{array}{c}40(34 / 6) \\
11\end{array}$ \\
\hline Median time to recurrence $(\mathrm{mo})[95 \% \mathrm{CI}]$ & $9(4-47)$ & N/A & $12(2.5-48.9)$ \\
\hline Overall survival rate & $66 \%$ at $3 y$ & $33.5 \%$ at $5 \mathrm{y}$ & $46.1 \%$ at $5 \mathrm{y}$ \\
\hline Median survival time (mo $[95 \% \mathrm{CI}]$ ) & N/A & $30(1.8-4.1)$ & 51.4 \\
\hline Predictors of LRs & Clinical $\mathrm{T}$ stage & N/A & $\begin{array}{c}\text { Age } \\
\text { Clinical stage }\end{array}$ \\
\hline Predictors of DRs & $\begin{array}{c}\text { Age } \\
\text { Tumor location }\end{array}$ & N/A & Age \\
\hline
\end{tabular}

$c C R$, Complete clinical response; $d C R T$, definitive chemoradiotherapy; $E S C C$, esophageal squamous cell carcinoma; $L R s$, locoregional recurrences; $D R s$, distant recurrences; $N / A$, not applicable.

dCRT-treated patients, it is reasonable to hypothesize that a significant proportion of cCR patients after dCRT could bear unidentified residual disease. In this context, high LR rates are not surprising.

Owing to the common occurrence of LRs even in patients with no evidence of disease (ie, cCR) after dCRT, a shift from dCRT to nCRT followed by scheduled resection should be carefully considered for all medically fit patients. The importance of completing a thorough imaging study to exclude persistent disease and close surveillance protocols tailored to early identification of recurrences are of paramount importance in all patients who are unwilling to undergo esophagectomy and choose dCRT as their first-line treatment.

Despite LR being the predominant recurrence pattern, the question as to whether the use of endoscopic surveillance could result in earlier cancer detection remains open. Recently, studies focusing on the distribution of residual cancer in post-nCRT patients have shown that most of the initially undetected tumors are located in the esophagus, being more commonly localized close to the luminal side (ie, in the mucosa or the submucosal layer) ${ }^{18,19}$ In this scenario, luminal regrowth - and not recurrence in the circumferential margin side-is expected to be the most common LR pattern. If this is the case, such lesions may be detectable through close endoscopic surveillance before becoming unresectable. A trial on the potential usefulness of close endoscopic surveillance and surgery as needed in cCR patients following nCRT is currently being conducted. ${ }^{20}$ We assume that the same phenomenon could be valid in patients treated with dCRT patients, although further investigations in support of this hypothesis are required. An observation that indirectly supports the above-mentioned hypothesis is the poor salvage surgery rate after LR $(38.2 \%)$ observed in our patients who did not routinely undergo an upper endoscopic surveillance program. Only CT imaging was routinely used for surveillance in our study, whereas upper endoscopy was used only when clinical symptoms of recurrent dysphagia were present. A high proportion of LR patients were judged to be unresectable (owing to tumor infiltration into adjacent organs) based on the presence of advanced local disease on CT images. In these circumstances, only second-line chemotherapy can be offered, and this option is less likely to produce positive results. ${ }^{21}$ Even among patients who received surgery, R0 resection-the most important determinant of survival after salvage esophagectomy-was achieved only in $46.1 \%$ of cases, a percentage significantly lower than those reported $(81 \%-87 \%)$ in previous studies. $^{22-24}$ In this context, it is not surprising that survival after recurrence was equally poor in patients with both LRs and DRs.

Based on our data (Figure 3) and the National Comprehensive Cancer Network guidelines, ${ }^{13}$ we suggest that patients who achieve cCR after dCRT should be closely monitored by upper endoscopy examinations performed at least every 3 months for the first 2 years (during which $95 \%$ of LRs occur). Moreover, we suggest performing CT 
or PET/CT with contrast medium at least every 6 months for the first 4 years (during which $90 \%$ of DRs occur) and on an annual basis thereafter (Table E2). ${ }^{25}$ Such a strict follow-up schedule would enable detection of recurrences at a relatively early stage, ultimately allowing the implementation of salvage resection.

In addition to refining surveillance protocols, the stratification of patients according to the risk of recurrence may further improve their outcomes by referring highrisk patients to additional local treatments. It has been previously shown that the percentage by which the maximum PET standardized uptake value is reduced, as well as the appearance on endoscopy, are associated with the risk of local residual disease in $\mathrm{cCR}$ patients who are treated with neoadjuvant CRT and surgery. ${ }^{11,26}$ In our study, we identified pretreatment cancer clinical stage as the only independent risk factor for LR after dCRT. This variable may guide the selection of patients at high risk for LR who may benefit from immediate surgery (rather than a watchful watching strategy). Future clinical trials are required to establish whether switching from watchful waiting to immediate surgery could be a suitable strategy for patients at risk of LR who are medically fit for surgery.

In contrast to the well-documented survival benefit conferred by salvage esophagectomy in the treatment of LRs occurring after dCRT, the role of surgical removal of solitary DRs after dCRT remains unclear. ${ }^{22,23}$ In our study, the postrecurrence OS of the 3 patients who underwent curative resection of a distant recurrence was 663 days (after brain tumor resection), 910 days (after cervical neck lymph node resection), and 911 days (after lung tumor resection), being overall acceptable. Several small, retrospective studies have shown that the surgical resection of oligometastases to the lung, brain, and neck lymph nodes may improve clinical outcomes. ${ }^{27-30}$ Future larger studies are needed before more definitive conclusions can be made.

Several caveats should be considered when interpreting our findings. First, our research was limited by its singlecenter, retrospective nature, as well as a prolonged enrollment period (13 years). Although we were able to perform a clinical re-review of all patients who achieved cCR, a large proportion of patients were treated during the early 2000s when PET scans were not frequently performed. Consequently, the occurrence of some misclassifications cannot be ruled out. We also acknowledge that the small number of recurrences could have precluded the detection of small but clinically important associations between the risk factors and the clinical outcomes. Finally, all participants had a diagnosis of ESCC; therefore, we are unable to generalize our results to patients with esophageal adenocarcinoma. Additional studies are required to replicate and validate our data in independent clinical settings and to optimize the follow-up schedules of patients who achieve cCR following dCRT.

\section{CONCLUSIONS}

Disease recurrence occurs in $50 \%$ of ESCC patients who achieve cCR following dCRT, with LR being the most common pattern. LRs occur significantly earlier than DRs, with $95 \%$ of the former diagnosed within 2 years. An advanced pretreatment clinical stage is an independent risk factor for LRs (Video 1).

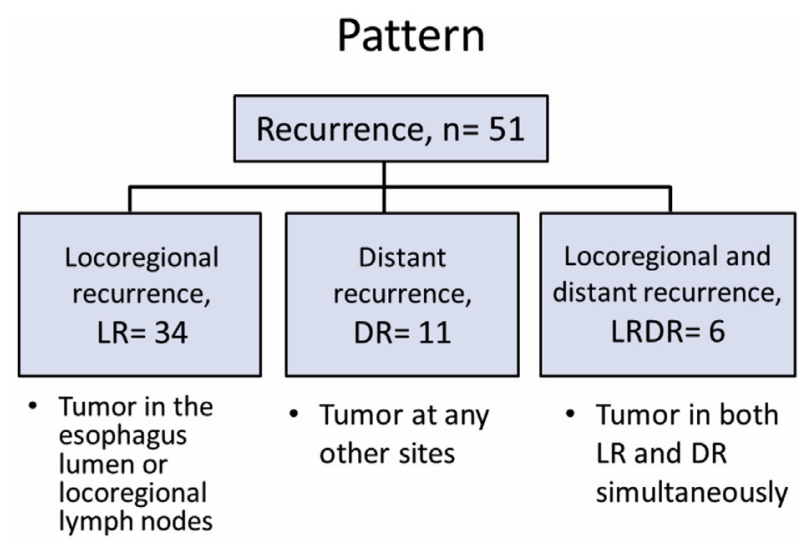

VIDEO 1. Definitive chemoradiotherapy (dCRT) is an alternative curative nonsurgical treatment option for patients with locally advanced esophagus squamous cell carcinoma (ESCC) who are medically unfit for surgery or unwilling to undergo operation. After dCRT, patients underwent close surveillance for clinical complete response (cCR), and treatment was provided in case of recurrences. Despite no evidence of residual cancer in patients who achieved cCR, tumor recurrences rates were high. We therefore investigated the timing, patterns, and predictors of recurrences in ESCC patients who achieved cCR following dCRT. Case records were reviewed and a total of 102 patients who attained cCR after dCRT were examined. Ultimately, 51 patients $(50 \%)$ showed recurrences, whereas the remaining $51(50 \%)$ did not. The cumulative 1-, 3-, and 5-year recurrence rates were $35 \%$, $46 \%$, and $50 \%$, respectively. The median overall survival was significantly lower in patients who experienced recurrences (18.4 months) compared to those who did not $(101$ months; $P<.0001)$. We then categorized the 51 cases of recurrence into locoregional ( $\mathrm{LR}=34$ cases) versus distant $(\mathrm{DR}=11$ cases $)$ versus locoregional plus distant recurrences (LRDR $=6$ cases). We found that LR was the most common recurrence pattern. The mean time to LR recurrence (281.4 days) was significantly shorter than the time to DR recurrence (643.6 days), with $95 \%$ of all LRs being diagnosed within 2 years $(P=.006)$. Cox regression analysis revealed that clinical stage was the only independent predictor of LR. We therefore recommend that patients who achieve cCR should be closely followed by using upper endoscopy at least every 3 months during the first 2 years. Because $90 \%$ of DRs occurred within the first 4 years, contrastenhanced computed tomography or positron emission tomography examinations should be performed at least every 6 months during this time period. $L R$, Local recurrence; $D R$, distant recurrence; $L R D R$, combined locoregional and distant recurrence. Video available at: http://www. jtcvsonline.org/article/S0022-5223(17)33060-X/fulltext. 


\section{Conflict of Interest Statement}

Authors have nothing to disclose with regard to commercial support.

\section{References}

1. Siegel RL, Miller KD, Jemal A. Cancer statistics, 2015. CA Cancer J Clin. 2015; 65:5-29.

2. Stahl M, Stuschke M, Lehmann N, Meyer HJ, Walz MK, Seeber S, et al. Chemoradiation with and without surgery in patients with locally advanced squamous cell carcinoma of the esophagus. J Clin Oncol. 2005;23:2310-7.

3. Bedenne L, Michel P, Bouché O, Milan C, Mariette C, Conroy T, et al. Chemoradiation followed by surgery compared with chemoradiation alone in squamous cancer of the esophagus: FFCD 9102. J Clin Oncol. 2007;25:1160-8.

4. Gwynne S, Hurt C, Evans M, Holden C, Vout L, Crosby T. Definitive chemoradiation for oesophageal cancer - a standard of care in patients with nonmetastatic oesophageal cancer. Clin Oncol. 2011;23:182-8.

5. Ishikura S, Nihei K, Ohtsu A, Boku N, Hironaka S, Mera K, et al. Long-term toxicity after definitive chemoradiotherapy for squamous cell carcinoma of the thoracic esophagus. J Clin Oncol. 2003;21:2697-702.

6. Ishihara R, Yamamoto S, Iishi H, Takeuchi Y, Suimoto N, Higahino K, et al. Factors predictive of tumor recurrence and survival after initial complete response of esophageal squamous cell carcinoma to definitive chemoradiotherapy. Int J Radiat Oncol Biol Phys. 2010;76:123-9.

7. Adenis A, Tresch E, Dewas S, Romano O, Messager M, Aela E, et al. Clinical complete responders to definite chemoradiation or radiation therapy for oesophageal cancer: predictors of outcome. BMC Cancer. 2013;13:1.

8. Suzuki A, Xiao L, Hayashi Y, Macapiniac HA, Welsh J, Lin SH, et al. Prognostic significance of baseline positron emission tomography and importance of clinical complete response in patients with esophageal or gastroesophageal junction cancer treated with definitive chemoradiotherapy. Cancer. 2011;117:4823-33.

9. Tahara M, Ohtsu A, Hironaka S, Boku N, Ishikura S, Miyat Y, et al. Clinical impact of criteria for complete response (CR) of primary site to treatment of esophageal cancer. Jpn J Clin Oncol. 2005;35:316-23.

10. Wu AJ, Goodman KA. Clinical tools to predict outcomes in patients with esophageal cancer treated with definitive chemoradiation: are we there yet? J Gastrointest Oncol. 2014;6:53-9.

11. Molena D, Sun H, Badr A, Mungo B, Sarkaria I, Adusumilli P, et al. Clinical tools do not predict pathological complete response in patients with esophageal squamous cell cancer treated with definitive chemoradiotherapy. Dis Esophagus. 2014;27:355-9.

12. Miyata H, Yamasaki M, Takiguchi S, Nakajima K, Fujiwara Y, Konishi K, et al. Prognostic value of endoscopic biopsy findings after induction chemoradiotherapy with and without surgery for esophageal cancer. Ann Surg. 2011; 253:279-84.

13. Ajani JA, D'Amico TA, Almhanna K, Bentrem DJ, Besh S, Chao J, et al. Esophageal and esophagogastric junction cancers, version 1.2015. J Natl Compr Canc Netw. 2015;13:194-227.

14. Oken MM, Creech RH, Tormey DC, Horton J, Davis TE, McFadden ET, et al. Toxicity and response criteria of the Eastern Cooperative Oncology Group. Am J Clin Oncol. 1982;5:649-56.

15. Charlson ME, Pompei P, Ales KL, MacKenzie CR. A new method of classifying prognostic comorbidity in longitudinal studies: development and validation. $J$ Chronic Dis. 1987;40:373-83.
16. Eisenhauer E, Therasse P, Bogaerts J, Schwartz LH, Sargent D, Ford R, et al. New response evaluation criteria in solid tumours: revised RECIST guideline (version 1.1). Eur J Cancer. 2009;45:228-47.

17. De Wreede LC, Fiocco M, Putter H. The mstate package for estimation and pre diction in non-and semi-parametric multi-state and competing risks models. Comput Method Prog Biomed. 2010;99:261-74.

18. Shapiro J, ten Kate FJ, van Hagen P, Biermann K, Wijnhoven BP, van Lanschot JJB. Residual esophageal cancer after neoadjuvant chemoradiotherapy frequently involves the mucosa and submucosa. Ann Surg. 2013;258: 678-89.

19. Chao Y-K, Tsai C-Y, Chang H-K, Tseng C-K, Liu Y-H, Yeh C-JA. Pathological study of residual cancer in the esophageal wall following neoadjuvant chemoradiotherapy: focus on esophageal squamous cell carcinoma patients with false negative preoperative endoscopic biopsies. Ann Surg Oncol. 2015; 22:1-6.

20. Noordman BJ, Shapiro J, Spaander MC, Krishnadath KK, van Laarhover HW, van Berge Henegouwen MI, et al. Accuracy of detecting residual disease after CROSS neoadjuvant chemoradiotherapy for esophageal cancer (preSANO trial): rationale and protocol. JMIR Res Protoc. 2015;4:e79.

21. Thallinger CMR, Raderer M, Hejna M. Esophageal cancer: a critical evaluation of systemic second-line therapy. J Clin Oncol. 2011;29:4709-14.

22. Farinella E, Safar A, Nasser HA, Bouazza F, Liberale G, Paesmans M, et al. Salvage esophagectomy after failure of definitive radiochemotherapy for esophageal cancer. J Surg Oncol. 2016;114:833-7.

23. Markar S, Gronnier C, Duhamel A, Pasquer A, Therauax J, du Riec MC, et al. Salvage surgery after chemoradiotherapy in the management of esophageal cancer: is it a viable therapeutic option? J Clin Oncol. 2015;33:3866-73.

24. Wang S, Tachimori Y, Hokamura N, Igaki H, Nakazato H, Kishino T. Prognostic analysis of salvage esophagectomy after definitive chemoradiotherapy for esophageal squamous cell carcinoma: the importance of lymphadenectomy. J Thorac Cardiovasc Surg. 2014;147:1805-11.

25. Guo H, Zhu H, Xi Y, Zhang B, Li L, Huag Y, et al. Diagnostic and prognostic value of $18 \mathrm{~F}-\mathrm{FDG}$ PET/CT for patients with suspected recurrence from squamous cell carcinoma of the esophagus. J Nuc Me. 2007;48:1251-8.

26. Fang HY, Chao YK, Chang HK, Tseng CK, Liu YH. Survival outcomes of consolidation chemoradiotherapy in esophageal cancer patients who achieve clinical complete response but refuse surgery after neoadjuvant chemoradiotherapy. Dis Esophagus. 2017;30:1-8.

27. Yano M, Takachi K, Doki Y, Miyashiro I, Kishi K, Noura S, et al. Prognosis of patients who develop cervical lymph node recurrence following curative resection for thoracic esophageal cancer. Dis Esophagus. 2006; 19:73-7.

28. Song Z, Lin B, Shao L, Zhang Y. Brain metastases from esophageal cancer: clinical review of 26 cases. World Neurosurg. 2014;81:131-5.

29. Chen F, Sato K, Sakai H, Mihahara R, Bando T, Okubo K, et al. Pulmonary resection for metastasis from esophageal carcinoma. Interact Cardiovasc Thorac Surg. 2008; 7:809-12.

30. Kozu Y, Sato H, Tsubosa Y, Ogawa H, Yasui H, Kondo H. Surgical treatment for pulmonary metastases from esophageal carcinoma after definitive chemoradiotherapy: experience from a single institution. J Cardiothorac Surg. 2011; $6: 135$.

Key Words: esophageal cancer, definitive chemoradiotherapy, clinical complete response, recurrence 


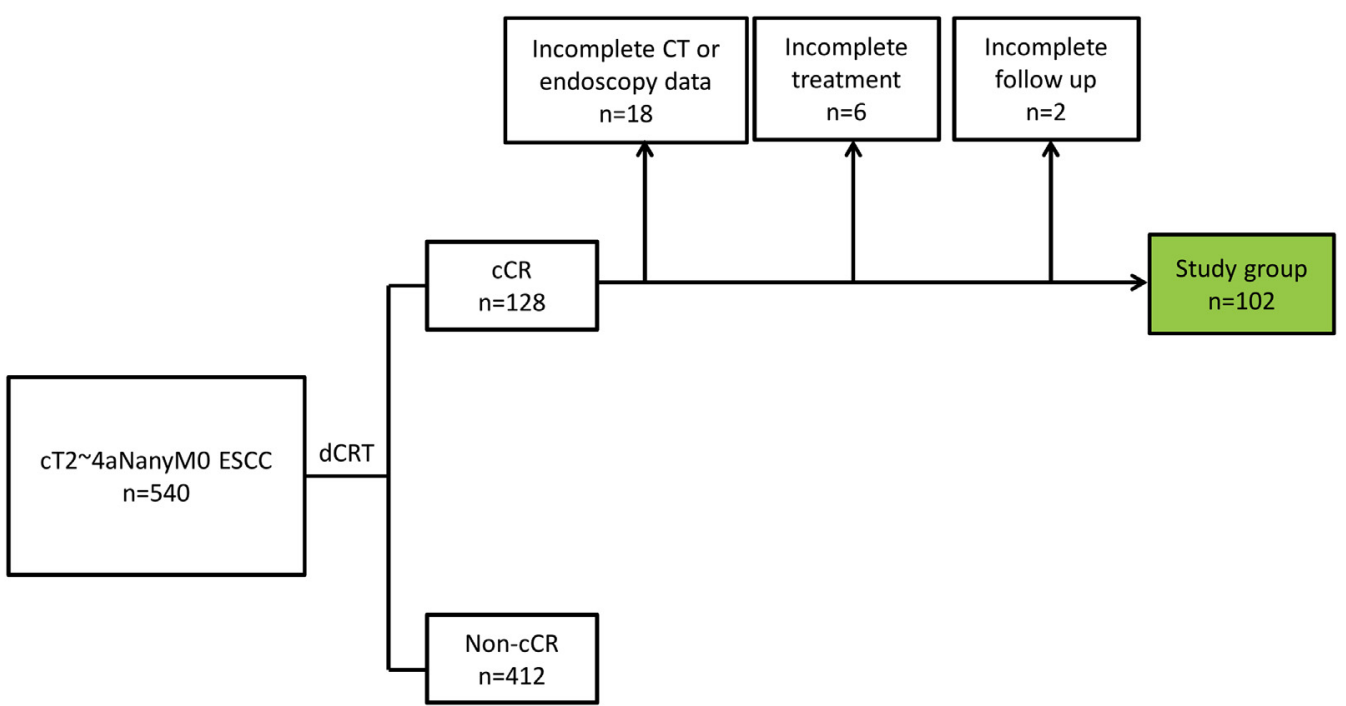

FIGURE E1. Flow diagram of the study. $C T$, Computed tomography; $c C R$, clinical complete response; $E S C C$, esophageal squamous cell carcinoma; $d C R T$, definitive chemoradiotherapy.

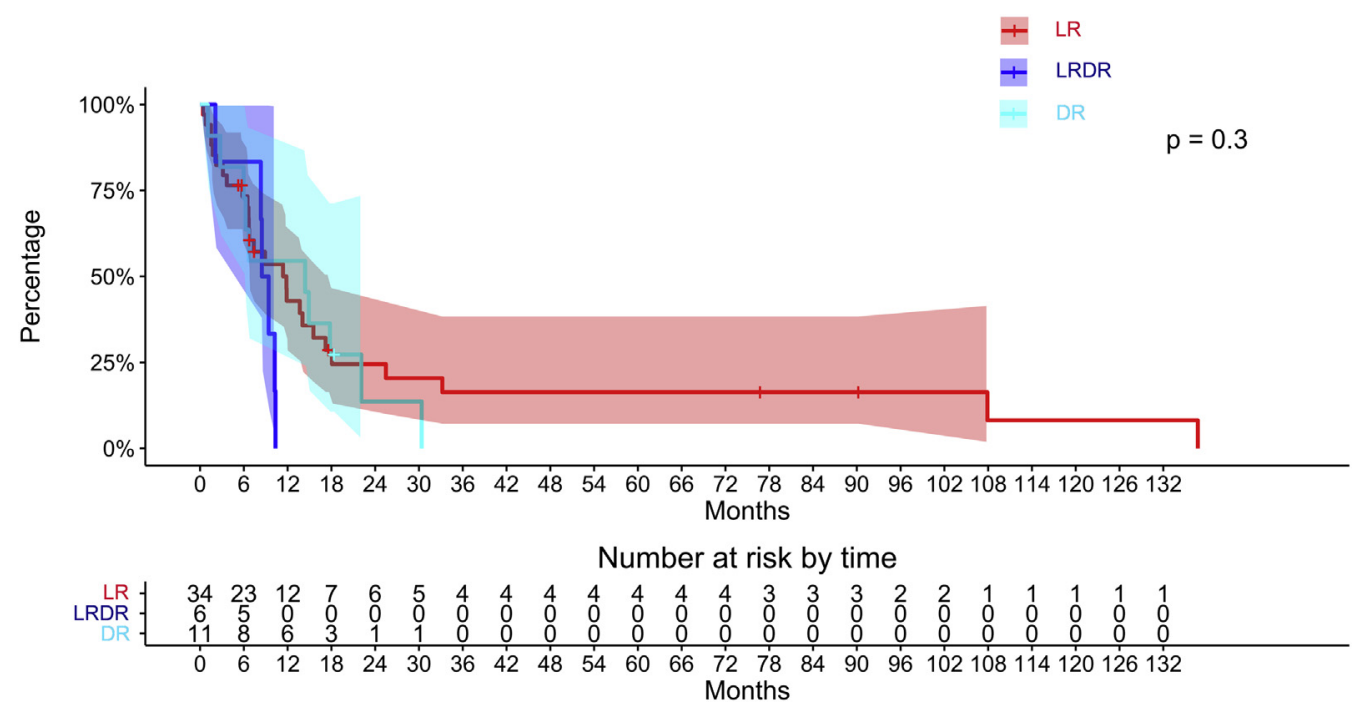

FIGURE E2. Overall survival after locoregional recurrences (LRs), distant recurrences (DRs), and combined locoregional and distant recurrences (LRDRs). 
TABLE E1. Results of univariate and multivariable Cox regression analyses for risk factors of distant recurrence

\begin{tabular}{|c|c|c|}
\hline Risk factor & $\begin{array}{c}\text { Univariate } \\
\text { Hazard ratio }(95 \% \\
\text { confidence interval) }\end{array}$ & $P$ value \\
\hline Age (y) & $0.951(0.918-0.996)$ & .033 \\
\hline \multicolumn{3}{|l|}{ Sex } \\
\hline Male & Reference & .248 \\
\hline Female & $2.345(0.535-10.275)$ & \\
\hline \multicolumn{3}{|l|}{ ECOG scale } \\
\hline 0 & Reference & \\
\hline 1 & $1.498(0.195-11.512)$ & .698 \\
\hline 2 & $0.414(0.026-6.714)$ & .535 \\
\hline Tumor length (cm) & $0.964(0.805-1.156)$ & .695 \\
\hline \multicolumn{3}{|l|}{ Location } \\
\hline Upper third & Reference & \\
\hline Middle third & $0.718(0.252-2.047)$ & .535 \\
\hline Lower third & $1.18(0.289-4.329)$ & .871 \\
\hline \multicolumn{3}{|l|}{ Clinical $\mathrm{T}$ stage } \\
\hline 2 & Reference & .291 \\
\hline 3 or 4 & $1.961(0.561-6.852)$ & \\
\hline \multicolumn{3}{|l|}{ Clinical stage } \\
\hline II & Reference & .096 \\
\hline III & $3.519(0.798-15.513)$ & \\
\hline \multicolumn{3}{|c|}{ Radiotherapy dose (cGy) } \\
\hline$\leq 6000$ & Reference & .980 \\
\hline$>6000$ & $10.26(0.136-7.746)$ & \\
\hline \multicolumn{3}{|l|}{ Chemotherapy } \\
\hline $\mathrm{PF}$ regimen & Reference & .212 \\
\hline PC regimen & $3.678(0.476-28.421)$ & \\
\hline
\end{tabular}

$E C O G$, Eastern Cooperative Oncology Group; $P F$, cisplatin + 5-fluorouracil; $P C$, paclitaxel + carboplatin.

TABLE E2. Proposed follow-up protocol for patients with clinical complete response after definitive chemoradiotherapy

\begin{tabular}{lcc}
\hline Month of follow-up & Upper endoscopy & CT or PET/CT \\
\hline 3 & + & + \\
6 & + & \\
\hline 9 & + & + \\
12 & + & + \\
15 & + & \\
18 & + & + \\
\hline 21 & + & + \\
24 & + & + \\
\hline 30 & & + \\
36 & & + \\
42 & & + \\
48 & & + \\
\hline 60 & & + \\
72 & & + \\
\hline
\end{tabular}

CT, Computed tomography; PET, positron-emission tomography. 